\title{
A charge-reversible nanocarrier using PEG-PLL (-g-Ce6, DMA)-PLA for photodynamic therapy
}

This article was published in the following Dove Press journal:

International Journal of Nanomedicine

24 August 2017

Number of times this article has been viewed

\section{Chaemin Lim' \\ Taehoon Sim' \\ Ngoc Ha Hoang ${ }^{2}$ \\ Chan Eun Jung' \\ Eun Seong Lee ${ }^{3}$ \\ Yu Seok Youn ${ }^{4}$ \\ Kyung Taek Oh'}

'Department of Pharmaceutical Sciences, College of Pharmacy, Chung-Ang University, Heukseokdong, Dongjak-gu, Seoul, South Korea; ${ }^{2}$ Department of Pharmaceutics, $\mathrm{Ha}$ Noi University of Pharmacy, Ha Noi, Vietnam; ${ }^{3}$ Division of Biotechnology, The Catholic University of Korea, Gyeonggi-do, Bucheon, ${ }^{4}$ Department of Pharmaceutical Sciences, School of Pharmacy, Sungkyunkwan University, Suwon, South Korea
Correspondence: Kyung Taek Oh Department of Pharmaceutical Sciences, College of Pharmacy, Chung-Ang University, 84 Heukseok-ro, Dongjak-gu, Seoul 06974, South Korea

Tel/fax +82 28245617

Email kyungoh@cau.ac.kr
Abstract: A polyelectrolyte nanoparticle composed of PEG-PLL(-g-Ce6, DMA)-PLA was developed for nanomedicinal application in photodynamic therapy. These nanoparticles formed stable aggregates through the hydrophobic interaction of poly(lactic acid) and demonstrated $\mathrm{pH}-$ dependent behaviors such as surface charge conversion and enhanced cellular uptake at acidic $\mathrm{pH}$, resulting in improved phototoxicity. In vivo animal imaging revealed that the prepared PEG-PLL(-g-Ce6, DMA)-PLA nanoparticles effectively accumulated at the targeted tumor site through enhanced permeability and retention effects. Reversible surface charge for PEG-PLL (-g-Ce6, DMA)-PLA nanoparticles allows the nanoparticles to escape the immune system and concentrate on the tumor tissue. Tumor growth in the nude mice treated with the nanoparticles decreased significantly and the hydrophobic interaction in the poly(lactic acid) block could allow the incorporation of multiple drugs. Therefore, the PEG-PLL(- $g$-Ce6, DMA)-PLA nanoparticles could have considerable potential as a nanomedicinal platform for photodynamic therapy.

Keywords: apoptosis, chlorin e6, polyelectrolyte, $\mathrm{pH}$ sensitive, charge conversion, nanomedicine

\section{Introduction}

Photodynamic therapy (PDT) has recently attracted much attention in cancer treatment. ${ }^{1-3}$ PDT treatment is based on the administration of a photosensitizer, followed by illumination of cancer tissue using visible light of a specific wavelength. PDT allows local and precisely targeted cancer treatment and, unlike radiation, can be repeated many times at the same site. ${ }^{4,5}$ However, many photosensitizers are insoluble in water and, after intravenous administration, do not specifically localize in tumor cells, resulting in some toxicity to healthy cells and tissues. ${ }^{6-9}$ Therefore, nanoparticles are required to solubilize and deliver photosensitizers to the tumor site by passive or active targeting. Recently, photosensitizer formulations using polymeric nanoparticles have been intensively investigated as promising PDT agents. ${ }^{10,11}$ In particular, environmentally responsive nanoparticles have attracted much attention in PDT for their potential to reduce toxicity, their enhanced permeability and retention (EPR) effects that allow passive targeting, and their ability to control the release of photosensitizers through tumor extracellular $\mathrm{pH}$ or endosomal $\mathrm{pH} .{ }^{12-14}$

In the present study, we prepared a surface charge-reversible, stable nanoplatform to maximize the therapeutic effects of PDT. It was reported that nanoparticles with a positive charge can enhance the cellular uptake of therapeutic agents through electrostatic interaction with the negatively charged cell membrane. ${ }^{15}$ However, in vivo biodistribution studies demonstrated that undesirable liver uptake could be increased for positively charged nanoparticles, likely due to phagocytosis by macrophages 
in the liver. ${ }^{16,17}$ On the other hand, negatively charged nanoparticles showed decreased liver uptake and increased delivery efficiency to the tumor site. ${ }^{18,19}$ Therefore, there is an urgent need to develop an environmentally responsive charge-reversible PDT agent to increase cellular uptake efficiency in in vitro conditions and to maximize delivery efficiency to the tumor site in in vivo conditions. In our previous study, we developed a stable nanoplatform composed of poly(ethylene glycol)-poly(L-lysine)-poly(lactic acid) (PEG-PLL-PLA) triblock copolyelectrolyte. ${ }^{17}$ In this study, a typical photosensitizer, Chlorin e6 (Ce6), and a pH-responsive 2,3-dimethyl maleic anhydride (DMA) moiety were conjugated to the lysine residue in PEG-PLL-PLA, resulting in PEG-PLL(-g-Ce6, DMA)-PLA. Chlorin e6 is one of the most widely used phototherapeutic agents and it was used as a model drug in this study for photodynamic therapy. DMA is one of the most widely used pH-responsive linkages for environmentally responsive drug delivery platforms. In acidic buffer conditions, the DMA moiety can be cleaved from the lysine residue, resulting in regeneration of the positive charge. ${ }^{20,21}$ Thus, its surface charge reversibility prolongs the blood circulation time of PEG-PLL(- $g$-Ce6, DMA)-PLA nanoparticles, and when they accumulate in tumor sites, their surface charge changes from negative to positive, resulting in enhanced cellular uptake and improved PDT efficacy. Additionally, the PDT agent contains a hydrophobic PLA block that could provide increased colloidal stability in in vivo conditions. The PEG-PLL(- $g-\mathrm{Ce} 6$, DMA)-PLA nanoparticles also have the potential to incorporate multiple drugs through hydrophobic interaction and can thus be used for combination drug therapy. In this study, to evaluate the therapeutic potential of PEG-PLL(- $g$-Ce6, DMA)-PLA nanoparticles in PDT treatment, we conducted nanoparticle analysis, in vitro studies, and in vivo studies.

\section{Materials and methods Materials}

Methoxy polyethylene glycol amine (mPEG-NH2, molecular weight [MW] 5,000), $\mathrm{N}^{6}$-Carbobenzyloxy-L-lysine, 3,6-dimethyl-1,4-dioxite-2,5-dione, DMA, stannous octoate (Tin[II]2-ethylhexanoate), 4-(dimethylamino)pyridine (DMAP), succinic anhydride, pyridine, triethylamine (TEA), $N$ hydroxysuccinimide (NHS), $N, N^{\prime}$-dicyclohexylcarbodiimide (DCC), 9,10-dimethylanthracene, trifluoroacetic acid (TFA), $33 \% \mathrm{HBr}$ in acetic acid, anhydrous 1,4-dioxane, dimethyl sulfoxide (DMSO)- $\mathrm{d}_{6}$, and anhydrous dimethylformamide (DMF) were purchased from Sigma-Aldrich (St Louis, MO, USA). Triphosgene was purchased from Alfa Aesar ${ }^{\circledR}$ Johnson
Matthey Korea (Seoul, South Korea). Dichloromethane and toluene were purchased from Honeywell Burdick \& Jackson ${ }^{\circledR}$ (Muskegon, MI, USA). Chlorin e6 (Ce6) was purchased from Frontier Scientific Inc. (Logan, UT, USA). All other chemicals used were of analytical grade. For cell culture, human cervical cancer KB cells were obtained from the Korean Cell Line Bank (KCLB, Seoul, South Korea). RPMI 1640 medium, fetal bovine serum (FBS), penicillin, and streptomycin were purchased from Welgene (Seoul, South Korea). Cell Counting Kit-8 (CCK-8) was obtained from Dojindo Molecular Technologies (Tokyo, Japan). PEG-PLL-PLA triblock copolyelectrolyte was prepared as described previously. ${ }^{17}$

\section{Synthesis of $\mathrm{pH}$-sensitive PEG-PLL (-g-Ce6, DMA)-PLA}

In our previous research, we synthesized the PEG-PLLPLA triblock copolyelectrolyte. ${ }^{17}$ Briefly, PEG-poly(Nebenzyloxycarbonyl-L-lysine) (PEG-PBLL) was synthesized by ring opening polymerization (ROP) of N-carboxy(Ne-benzyloxycarbonyl)-L-lysine anhydride (Lys-NCA) using amine PEG $(5,000 \mathrm{Da})$ as a macro initiator. Then, the PEG-PBLL was coupled with activated PLA using a DCC and NHS reaction, and the benzyl group of PBLL was removed using TFA and $\mathrm{HBr}$ in acetic acid. The repeating units for PEG, PLL, and PLA numbered 114, 15, and 69, respectively. The prepared PEG-PLL-PLA $(0.3 \mathrm{mmol})$ was reacted with activated Ce6 (0.6 mmol), DMA (4 mmol), pyridine $(0.1 \mathrm{~mL})$, and TEA $(0.1 \mathrm{~mL})$ in DMF $(10 \mathrm{~mL})$ at room temperature for $48 \mathrm{~h}$. To remove the unreacted Ce 6 and DMA, the resulting solution was dialyzed in a dialysis membrane (Spectra/Pore; molecular weight cutoff [MWCO] 3,500) against DMSO for 3 days and freeze-dried. The Ce6 conjugation was confirmed by the presence of an ${ }^{1} \mathrm{H}$ NMR (DMSO-d6; Cambridge Isotope Lab. Inc., Tewksbury MA, USA) peak at $\delta 6.0-6.5$ and the physicochemical characterization of the Ce6-conjugated triblock copolyelectrolyte. The DMA conjugation was confirmed by the presence of an ${ }^{1} \mathrm{H}$ NMR peak at $\delta 1.8-2.0$. The conjugation efficiency of Ce6 into triblock copolymers was $50 \%$.

\section{Preparation of $\mathrm{pH}$-sensitive PEG- PLL(-g-Ce6 and DMA)-PLA micelles and measurement of $\mathrm{Ce} 6$ in the micelles}

PEG-PLL(-g-Ce6 and DMA)-PLA micelles (CDTM) were prepared using a membrane dialysis method. ${ }^{22,23}$ The PEG-PLL(-g-Ce6 and DMA)-PLA (10 mg) was dissolved in $2 \mathrm{~mL}$ of DMF, and the DMF solution was added dropwise into an aqueous solution with vigorous stirring. The 
solution was then transferred into a dialysis bag (MWCO $3,500)$ to dialyze against PBS ( $\mathrm{pH} 7.4,1 \times)$ for $24 \mathrm{~h}$. The concentration of Ce6 in CDTM was analyzed in DMF using a Labentech UV-Vis spectrophotometer (Incheon, South Korea) at $663 \mathrm{~nm}$. The calibration curve was obtained with $\mathrm{Ce} 6 / \mathrm{DMF}$ solutions with different $\mathrm{Ce} 6$ concentrations. The loading efficiency of Ce6 in CDTM calculated by the following equation was ca $94.6 \%$.

$$
\begin{aligned}
& \text { Loading efficiency }(\%) \\
& =\frac{\text { Weight of Ce } 6 \text { in micelles }}{\text { Weight of Ce } 6 \text { fed initially }} \times 100
\end{aligned}
$$

\section{Particle size and zeta potential measure- ment using dynamic light scattering}

The effective hydrodynamic diameters $\left(\mathrm{D}_{\text {eff }}\right)$ and zeta potentials of the nanoparticles were measured by photon correlation spectroscopy using a Zetasizer Nano-ZS (Malvern Instruments, Worcestershire, UK) equipped with the multiangle sizing option (BI-MAS). For characterization of CDTM at different $\mathrm{pHs}$, the prepared micelles were exposed to a specific $\mathrm{pH}$ condition (PBS $1 \times, \mathrm{pH}$ 5.5-8.5) and the $\mathrm{pH}$ of each buffered solution was finally adjusted by a $\mathrm{pH}$ meter before use. The average particle size and zeta potential values were calculated from three measurements performed on each sample $(n=3)$.

\section{Fluorescence and optical images of CDTM and free $\mathrm{Ce} 6$}

Fluorescence images of wells containing free $\mathrm{Ce} 6$ and CDTM in PBS at different $\mathrm{pH}$ were obtained using a fluorescence labeled organism bioimaging instrument (FOBI) fluorescence live imaging system (IFLIS; NeoScience, Suwon, South Korea). The fluorescence intensity (FI) of each well was calculated and plotted using equivalent $\mathrm{Ce} 62 \mu \mathrm{g} / \mathrm{mL}$. The UV-Vis spectra of CDTM and free $\mathrm{Ce} 6$ in distilled water were obtained using a UV-Vis spectrophotometer (Incheon, South Korea).

\section{Singlet oxygen generation}

The generation of singlet oxygen from free $\mathrm{Ce} 6$ and CDTM in PBS at pH 5.5 and 7.4 was measured using 9,10-dimethylanthracene as an extremely fast chemical trap for singlet oxygen. ${ }^{24,25}$ Each sample at different $\mathrm{pH}$ was stabilized at room temperature for $4 \mathrm{~h}$, and 9,10-dimethylanthracene $(20 \mathrm{mmol})$ was then mixed and irradiated for $100 \mathrm{~s}$ at a light intensity of $5.2 \mathrm{~mW} / \mathrm{cm}^{2}$ using a $670-\mathrm{nm}$ laser source. When the 9,10-dimethylanthracene FI reached a plateau (after $1 \mathrm{~h}$ ), the change in FI (measured using a Scinco FS-2 fluorescence spectrometer at ex $360 \mathrm{~nm}$ and em 380-550 nm) in each sample was plotted by subtracting each sample FI from the full 9,10-dimethylanthracene FI.

\section{In vitro cellular uptake study}

The cellular uptake of $\mathrm{Ce} 6$ was studied using flow cytometry. $\mathrm{KB}$ cells were seeded onto six-well plates at a density of $4 \times 10^{5}$ cells/well. CDTM or free Ce6 in RPMI-1640 medium at pH 6.0 and 7.4, adjusted with $0.1 \mathrm{~N} \mathrm{NaOH}$ or $0.1 \mathrm{~N} \mathrm{HCl}$, was added to the medium-removed six-well plates and incubated for $4 \mathrm{~h}$. The cells were then washed three times with cold PBS, harvested, and subsequently analyzed with a FACSCalibur flow cytometer using Cell Quest Pro software (BD Biosciences, San Diego, CA, USA).

\section{Reactive oxygen species measurement}

Reactive oxygen species (ROS) production was determined by using DCFDA Cellular ROS detection Assay Kit (Abcam, Cambridge, UK). The KB cells were seeded in a 96-well plate and incubated for $24 \mathrm{~h}$. Then, the cells were treated with CDTM at different $\mathrm{pH}$ conditions for $4 \mathrm{~h}$ and re-incubated with $100 \mu \mathrm{L}$ of a $25 \mu \mathrm{M}$ DCFDA solution in the dark for $45 \mathrm{~min}$. Later, the cells were washed with $1 \times$ PBS followed by PDT treatment with different light exposure time $\left(5.2 \mathrm{~mW} / \mathrm{cm}^{2}, 670 \mathrm{~nm}\right.$, time: $100 \mathrm{~s}$ or $\left.200 \mathrm{~s}\right)$. Fluorescence intensity from the cells of each well was measured using a Scinco FS-2 fluorescence spectrometer with a $485 \mathrm{~nm}$ excitation filter and a $535 \mathrm{~nm}$ emission filter.

\section{In vitro phototoxicity}

KB cells in growth medium were seeded on 96-well plates at a density of $10^{4}$ cells/well $24 \mathrm{~h}$ prior to the phototoxicity test. CDTM and free Ce6 in RPMI-1640 medium at different $\mathrm{pH}$ were added and incubated for $4 \mathrm{~h}$. The cells were washed three times with PBS and irradiated for $100 \mathrm{~s}$ at a light intensity of $5.2 \mathrm{~mW} / \mathrm{cm}^{2}$ using a $670 \mathrm{~nm}$ fibercoupled laser system and then further incubated for $24 \mathrm{~h}$ in RPMI-1640. Cell viability was assessed using the CCK assay. For this, $90 \mu \mathrm{L}$ of fresh medium with $10 \mu \mathrm{L}$ of CCK solution was added to each well, and the plate was incubated for an additional $3 \mathrm{~h}$. The absorbance of each well was read on a Flexstation 3 microplate reader (Molecular Devices, Sunnyvale, CA, USA) at a wavelength of $450 \mathrm{~nm}$.

\section{FITC-annexin V/propidium iodide double staining}

The dead cell apoptosis kit with annexin V FITC and propidium iodide (PI) (V13242, Invitrogen) was used for detection 
of apoptosis and necrosis induced by PDT treatment. Cells were seeded in a six-well plate and incubated for $24 \mathrm{~h}$. Then, the cells were treated with CDTM (equivalent Ce6 $1 \mu \mathrm{g} / \mathrm{mL}$ ) for $4 \mathrm{~h}$ and irradiated at $660 \mathrm{~nm}$ with $5.2 \mathrm{~mW} / \mathrm{cm}^{2}$ for $100 \mathrm{~s}$. The cells were harvested at $1,3,6$, and $24 \mathrm{~h}$ after treatment with PDT and double stained with annexin V-FITC and PI. The cells were analyzed with a FACSCalibur flow cytometer using Cell Quest Pro software (BD Biosciences, San Diego, CA, USA).

\section{Wound healing assay}

For the in vitro scratch assay, KB cells were seeded in 24-well plates at a density of $10^{5}$ cells/well and allowed to grow to $\sim 90 \%$ confluence $(24 \mathrm{~h})$. The confluent cell monolayers were scratched using a sterile $200 \mu \mathrm{L}$ pipette tip. After being washed with PBS, cells were incubated for $4 \mathrm{~h}$ with fresh medium containing CDTM $(0.25 \mu \mathrm{g} / \mathrm{mL})$. The cells were again washed three times with PBS and irradiated for $100 \mathrm{~s}$ at a light intensity of $5.2 \mathrm{~mW} / \mathrm{cm}^{2}$ using a $670 \mathrm{~nm}$ fiber-coupled laser system. The healing of the scratched area was monitored using a model AE31 light microscope (Motic Hong Kong Ltd, Kowloon Bay, Hong Kong) incorporating Moticam Pro software (Motic). ${ }^{26,27}$

\section{Animal care}

In vivo studies were performed with 4-6-week-old female nude mice (BALB/c, nu/nu mice, Institute of Medical Science, Tokyo, Japan). The mice were maintained under the guidelines of a protocol approved by the Institutional Animal Care and Use Committee (IACUC) of Chung-Ang University of Korea. All experiments were approved by the IACUC and performed in compliance with the relevant laws and institutional guidelines (Animal protection law in Korea, National Institute of Health guidelines "Principles of laboratory animal care").

\section{In vivo fluorescence imaging}

For the in vivo animal experiments, $\mathrm{KB}$ tumor cells were introduced into female nude mice via subcutaneous injection of $1 \times 10^{6}$ cells suspended in PBS ( $\left.\mathrm{pH} 7.4\right)$. When the tumor volume reached $75 \mathrm{~mm}^{3}$, CDTM, Ce6-grafted PEG-PLL-PLA-based nanoparticles (CTM), or free Ce6 (equivalent Ce6 $0.75 \mathrm{mg} / \mathrm{kg}$ ) was injected intravenously through the tail vein. A FOBI fluorescence live imaging system (IFLIS; NeoScience) was used to obtain live fluorescence images of the mice. At $24 \mathrm{~h}$ post-injection, the nude mice were sacrificed, and the excised organs were analyzed.

\section{In vivo tumor inhibition}

$\mathrm{KB}$ tumor cells were administered to female nude mice via subcutaneous injection of $1 \times 10^{6}$ cells suspended in PBS ( $\mathrm{pH}$ 7.4). When the tumor volume reached $150 \mathrm{~mm}^{3}$, the mice were treated with PBS, free Ce6, CTM, or CDTM (equivalent Ce6 $1.5 \mathrm{mg} / \mathrm{kg}$ ). At $24 \mathrm{~h}$ post-injection of each sample, the tumor sites were locally illuminated for $20 \mathrm{~min}$ with a light intensity of $80 \mathrm{~mW} / \mathrm{cm}^{2}$ using a $670 \mathrm{~nm}$ laser source. For the in vivo tumor inhibition assay, the change in tumor volume was monitored over time. Tumor volume was calculated using the formula: length $\times(\text { width })^{2} / 2$. The body weight of each animal was also measured.

\section{Results}

\section{Synthesis and characterization of triblock copolymers}

In this study, we conjugated a pH-sensitive DMA moiety and photosensitive $\mathrm{Ce} 6$ to the amine residue in PEG-PLL-PLA, forming PEG-PLL(-g-Ce6, DMA)-PLA triblock copolymer, a surface charge-reversible photodynamic therapeutic agent. First, PEG-PLL-PLA was synthesized as described in our previous report. ${ }^{17}$ The number of monomer repeating units of PEG, PLA and PLL is 114, 15, and 69, respectively, and the total MW of the synthesized PEG-PLL-PLA was ca. 12,000 Da as determined by comparing the peak intensity ratio of the $\mathrm{CH} 2(\delta 3.4-3.6)$ of PEG with the $\alpha-\mathrm{CH}(\delta 4.2)$ of PLL

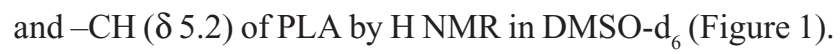
Second, the Ce6 and DMA were conjugated to lysine residue in the presence of NHS, DCC, TEA, and pyridine. The conjugation of $\mathrm{Ce} 6$ to PLL was confirmed by the presence of an ${ }^{1} \mathrm{H}$ NMR (DMSO-d $\mathrm{d}_{6}$, Cambridge Isotope Lab. Inc., Tewksbury, MA, USA) peak at $\delta 6.0-6.5 .{ }^{14}$ One molecule of Ce6 was conjugated to PEG-PLL-PLA triblock copolymer. The conjugation of DMA was confirmed by the presence of an ${ }^{1} \mathrm{H}$ NMR peak at $\delta 1.8-2.0 .^{28,29}$ The conversion of the amine group of PLL to DMA was $60 \%$, and the MW of the PEG-PLL(-g-Ce6, DMA)-PLA was ca. 13,700 Da (Figure 1). The triblock copolymer PEG-PLL-PLA-based nanoparticles (triblock micelle [TM]), CTM, and CDTM were prepared by the dialysis method. The TM, CTM, and CDTM showed particle sizes of 94, 112, and $94 \mathrm{~nm}$, respectively, with narrow size distributions. Even though the photosensitizer or $\mathrm{pH}$-sensitive moiety was grafted to the lysine residue in TM, there was no significant difference in particle size. When comparing the surface charge of the nanoparticles, only the CDTM exhibited a negative zeta potential, about $-14 \mathrm{mV}$. As expected, the zeta potential of CDTM was dramatically influenced by the carboxyl group of the DMA residue in the 


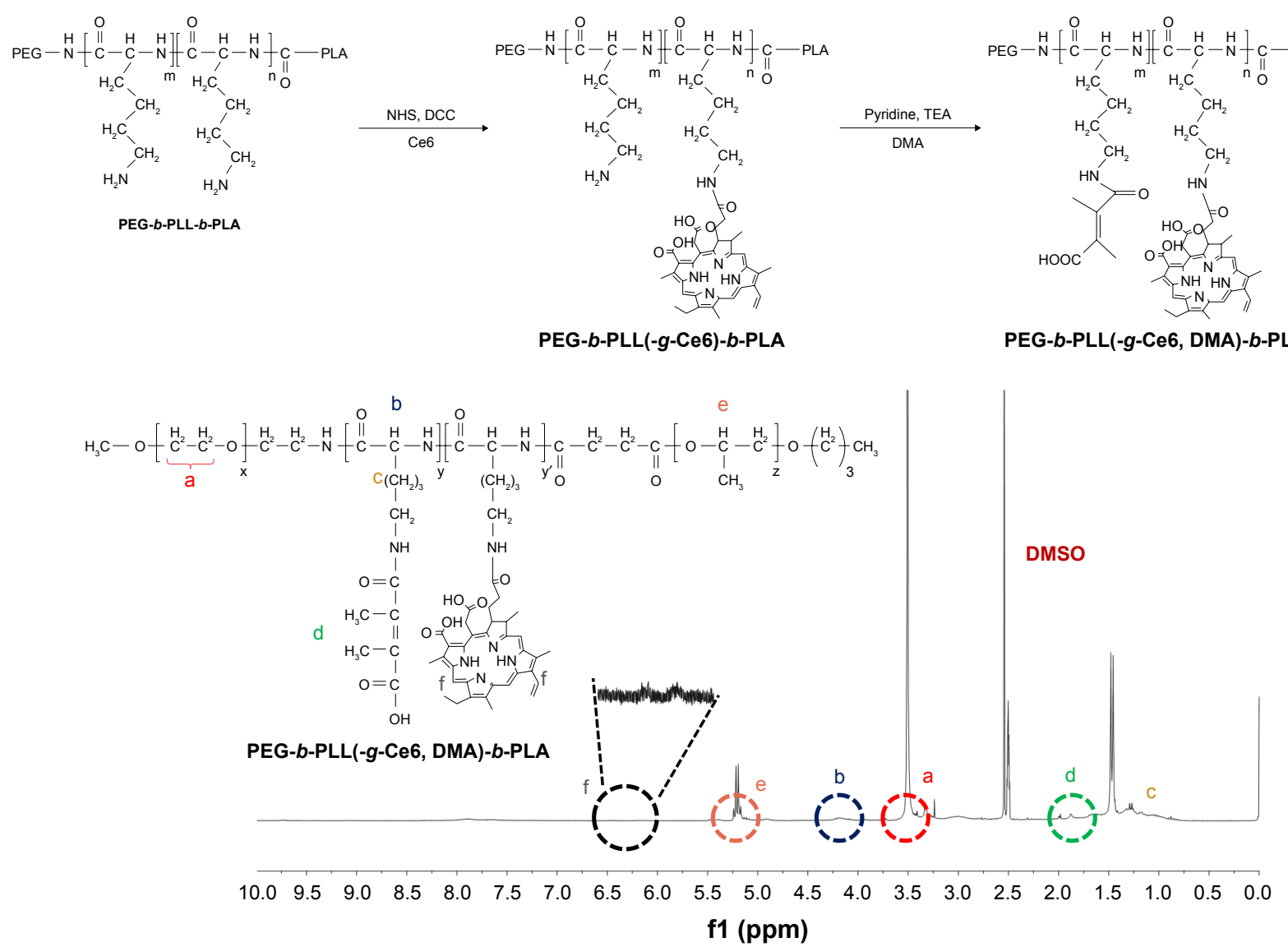

Figure I Overall scheme for the synthesis of PEG-b-PLL(-g-Ce6, DMA)-b-PLA and its validation by 'H NMR.

Notes: The Ce6 conjugation was confirmed by the presence of an 'H NMR (DMSO- $d_{6}$; Cambridge Isotope Lab. Inc., Tewksbury, MA, USA) peak at $\delta 6.0-6.5$ and the DMA conjugation was confirmed by the presence of an 'H NMR peak at $\delta$ I.8-2.0.

Abbreviations: Ce6, Chlorin e6; DCC, N,N'-dicyclohexylcarbodiimide; DMA, 2,3-dimethyl maleic anhydride; DMSO, dimethyl sulfoxide; NHS, N-hydroxysuccinimide; PEG, poly(ethylene glycol); PLA, poly(lactic acid); PLL, poly(L-lysine); TEA, triethylamine.

micelle structure. The zeta potential of TM was $+18 \mathrm{mV}$ in deionized water. When Ce6 was grafted to TM to form CTM, the surface charge decreased slightly to $+12 \mathrm{mV}$ (Table 1).

\section{Characterization of $\mathrm{pH}$-sensitive CDTM}

The surface charge of nanoparticles plays an important role in the efficiency of cellular uptake and biodistribution.

Table I Characteristics of the block copolymers

\begin{tabular}{|c|c|c|c|c|}
\hline Polymer & Code & $\begin{array}{l}\text { Size }^{a} \\
\left(D_{\text {eff }}, n m\right)\end{array}$ & PDI & $\begin{array}{l}\text { Zeta } \\
\text { potential } \\
(\mathrm{mV})^{\mathrm{a}}\end{array}$ \\
\hline PEG-b-PLL-b-PLA & TM & $94 \pm 4$ & $0.18 \pm 0.03$ & $+\mid 8.1 \pm 0.7$ \\
\hline PEG-b-PLL(-g-Ce6)-b-PLA & CTM & $112 \pm 14$ & $0.22 \pm 0.02$ & $+12 \pm 2$ \\
\hline $\begin{array}{l}\text { PEG-b-PLL(-g-Ce6, DMA)- } \\
b-P L A^{c}\end{array}$ & CDTM & $94 \pm 2.2$ & $0.20 \pm 0.02$ & $-14 \pm 2.5$ \\
\hline
\end{tabular}

Notes: aParticle size, PDI, and zeta potential were measured using DLS equipment; bMolar ratio between L-lysine and $\mathrm{Ce} 6=15: 1$; 'Molar ratio between L-lysine, Ce6, and DMA $=15: 1: 9$.

Abbreviations: CDTM, PEG-PLL(-g-Ce6 and DMA)-PLA micelles; Ce6, chlorin e6; CTM, PEG-PLL(-g-Ce6)-PLA micelles; $D_{\text {eff }}$ effective hydrodynamic diameters; DLS, dynamic light scattering; DMA, 2,3-dimethyl maleic anhydride; PDI, polydispersity index; PEG, poly(ethylene glycol); PLA, poly(lactic acid); PLL, poly(L-lysine).
Nanoparticles with a high positive charge exhibit strong affinity for negatively charged cell membranes, accounting for their high cellular uptake. However, nanoparticles with a positive charge also have higher phagocytic uptake and induce strong interaction with the reticuloendothelial system compared with negatively charged nanoparticles, which affects the biodistribution of the nanoparticles throughout the body. ${ }^{16,18,19}$ Thus, to take full advantage of the surface charge of nanoparticles in cellular uptake and biodistribution, we prepared $\mathrm{pH}$-sensitive surface charge-reversible nanoparticles (CDTM) and evaluated their colloidal properties at different $\mathrm{pH}$ conditions ( $1 \times \mathrm{PBS}, \mathrm{pH} 5.5-8.5)$.

The CDTM showed a pH-dependent change of particle size and zeta potential (Figure 2). It is well known that a small, absolute value of zeta potential implies rapid aggregation of nanoparticles. ${ }^{30-32}$ However, the CDTM has pegylated layer on the surface, which could be expected to prevent the aggregation of CDTM possibly. ${ }^{33,34}$ Across the range of $\mathrm{pH}$ conditions, the CDTM maintained its size of 90-120 nm 

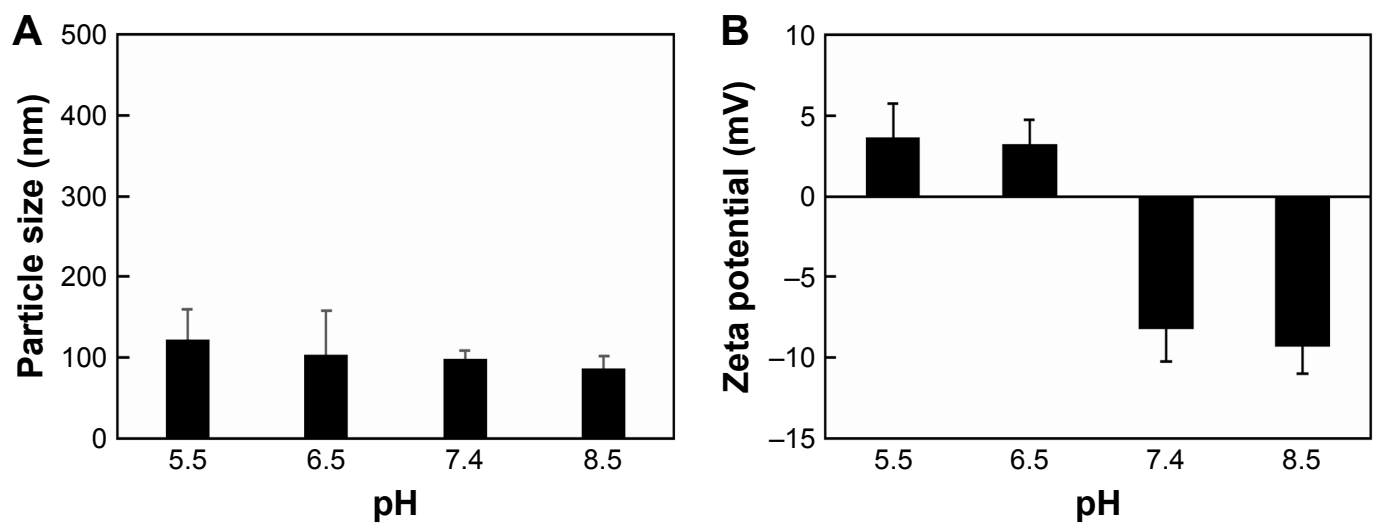

Figure 2 Characterization of CDTM. Change in (A) particle size and $(\mathbf{B})$ zeta potential of CDTM at different $\mathrm{pH}(\mathrm{n}=3)$.

Notes: The prepared micelles were exposed to a specific $\mathrm{pH}$ condition (PBS I×, $\mathrm{pH}$ 5.5-8.5) and the $\mathrm{pH}$ of each buffered solution was finally adjusted by a $\mathrm{pH}$ meter before use. The average particle size and zeta potential values were calculated from three measurements performed on each sample $(n=3)$.

Abbreviation: CDTM, PEG-PLL(-g-Ce6 and DMA)-PLA micelles.

(Figure 2A), which indirectly implies that the aggregation of CDTM has not happened. At pH 7.4 and 8.5, the zeta potential of CDTM was negative, -8 and -9 , reflecting the predominant conjugation of the DMA linkage to the lysine residue (Figure 2B). However, in acidic conditions, the zeta potential of CDTM became positive due to the cleavage of the amide bond between lysine and DMA, which could increase its cellular uptake efficiency. The morphology of CDTM at $\mathrm{pH} 7.4$ obtained from field emission scanning electron microscopy (FESEM) revealed regular spherical particles with a narrow size distribution (Figure S1A and B).

Ce6 is a derivate of chlorophyll a and contains three ionizable carboxylic groups. The presence of three carboxylic groups in Ce6 could affect its solubility in aqueous solution as $\mathrm{pH}$ conditions change. However, when $\mathrm{Ce} 6$ was conjugated to PEG-PLL-PLA, its solubility was maintained regardless of $\mathrm{pH}$ condition. ${ }^{35,36}$ Figure $3 \mathrm{~A}$ shows the change in FI of CDTM and free Ce6. When the $\mathrm{pH}$ decreased from 7.4 to 4.5 , the intensity of free $\mathrm{Ce} 6$ decreased due to aggregation, whereas CDTM showed a consistent FI over the whole $\mathrm{pH}$ range, demonstrating the conjugation of $\mathrm{Ce} 6$ to the lysine residue. Similarly, the production of singlet oxygen from Ce6 was downregulated in acidic conditions, which is comparable to the $\mathrm{pH}$-independent singlet oxygen generation of CDTM due to its stable formation over a broad $\mathrm{pH}$ range (Figure 3B). The absorbance spectrum of free Ce6 and CDTM in distilled water was measured using a UV-Vis spectrometer (Figure 3C). In distilled water, the free $\mathrm{Ce} 6$ was self-quenched by the aggregation of $\mathrm{Ce} 6$ molecules via hydrophobic and pi-pi interactions. However, the CDTM showed a high absorbance spectrum due to the improved water solubility of Ce6 after conjugation with the lysine residue. ${ }^{14,37}$

\section{In vitro evaluation of CDTM}

It is well known that nanoparticles employ multiple endocytic pathways to enter cells; especially, positively charged nanoparticles are well suited for endocytic cellular uptake because they can interact favorably with negatively charged cell membrane components. ${ }^{16,17}$ Thus, we used flow cytometry to examine the effects of CDTM surface charge reversibility on the cellular uptake of a drug in KB cells. As shown in Figure 4A, the mean FI of CDTM at pH 7.4 was about 57.5, but it increased to 97.7 at $\mathrm{pH} 6.0$, a remarkable enhancement in cellular uptake. The production of ROS by CDTM in in vitro conditions was also investigated (Figure 4B). Under the acidic condition, the level of ROS production was increased which can be attributed to the enhanced cellular uptake of CDTM at $\mathrm{pH}$ 6.0. The in vitro phototoxicity of the CDTM at different $\mathrm{pH}$ conditions was evaluated by a cell viability test. Cells were exposed to a series of equivalent concentrations of Ce 6 for $4 \mathrm{~h}$ and illuminated $\left(5.2 \mathrm{~mW} / \mathrm{cm}^{2}\right.$, $670 \mathrm{~nm}$ ) for $100 \mathrm{~s}$. As shown in Figure 4C, CDTM in acidic conditions achieved better phototoxicity effects than CDTM at pH 7.4, but there was no significant difference in the viability of cells treated with free $\mathrm{Ce} 6$ at different $\mathrm{pH}$ conditions. Thus, the positively charged CDTM enhanced the cellular uptake of the drug and thereby increased PDT efficacy. We also evaluated the level of induction of apoptosis and necrosis at different time points following light exposure. After CDTM-based PDT treatment, the KB cells were stained with annexin V FITC for apoptotic cells and with PI for necrotic cells. Over time, the number of apoptotic cells gradually increased from $5.4 \%$ to $27.5 \%$ and the number of late apoptotic-necrotic cells also significantly increased from $7.0 \%$ to $27.3 \%$. These results indicate that 

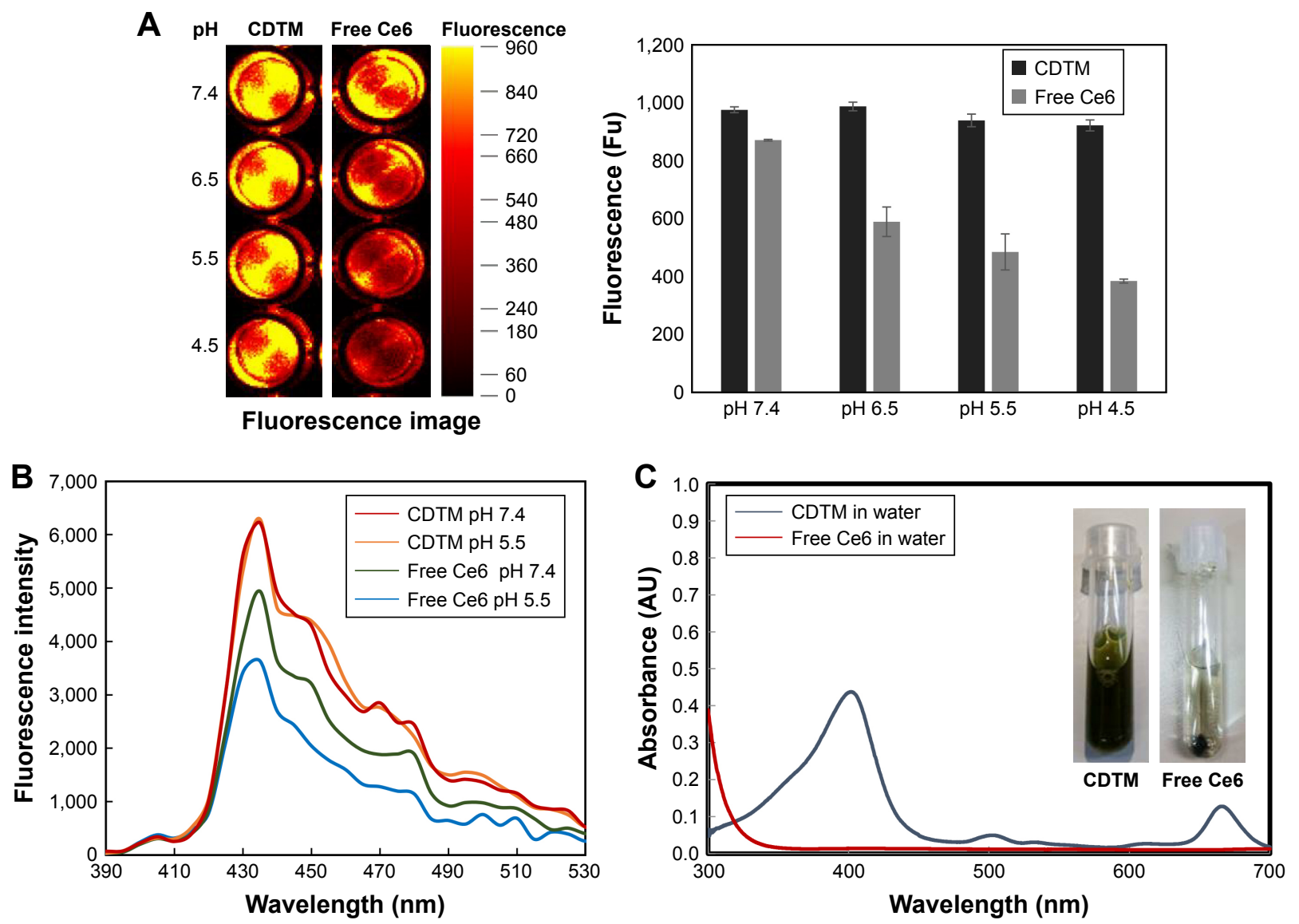

Figure 3 Physicochemical characteristics of free Ce6 and CDTM

Notes: (A) Fluorescence image of free Ce6 and CDTM in PBS based on an equivalent Ce6 concentration (2 $\mu \mathrm{g} / \mathrm{mL})$. (B) Singlet oxygen generation of free Ce6 and CDTM in PBS $(2 \mu \mathrm{g} / \mathrm{mL})$. (C) Optical image and UV-Vis spectrum scan of CDTM and free Ce6 in distilled water. When the pH decreased from 7.4 to 4.5 , the physicochemical characteristics of free $\mathrm{Ce} 6$ changed due to aggregation, whereas CDTM showed consistent physicochemical characteristics over the whole pH range, demonstrating the conjugation of $\mathrm{Ce} 6$ to the lysine residue.

Abbreviations: Ce6, chlorin e6; CDTM, PEG-PLL(-g-Ce6 and DMA)-PLA micelles; PBS, phosphate buffered saline.

CDTM-based PDT treatment could induce divergent cellular death pathways in KB cells (Figure 4D).

Tumor metastasis is a major cause of cancer-related mortality and remains the principal obstacle to successful chemotherapy. ${ }^{38,39}$ We used a cell scratch assay to evaluate how well CDTM inhibits cell migration. As shown in Figure 4E, after treatment with CDTM $(\mathrm{Ce} 6=0.25 \mu \mathrm{g} / \mathrm{mL})$ and illumination, the scratch remained, whereas the cells in the control group almost healed. It was reported that apoptosis, autophagy, and necrosis could play crucial roles in metastatic processes. In Figure 4D, we demonstrated that the CDTM could induce apoptosis and necrosis and it might be related to the inhibition of cell migration in KB cells. ${ }^{40,41}$

\section{In vivo tumor accumulation of CDTM}

Charge-reversible CDTM composed of PEG-PLL(-g-Ce6, DMA)-PLA was designed with the assumption that the Ce6-conjugated platform could be used as a diagnostic agent and as a therapeutic platform. ${ }^{42}$ The fluorescence imaging analysis was more widely applied in the preclinic research including theranostic approaches in order to non-invasively monitor their properties and biodistribution by fluorescence detection. ${ }^{43}$ In vivo, we expected the CDTM to circulate in the blood for a long time, escaping the immune system due to its slight negative charge and maintaining its shape through pegylation. ${ }^{19,44}$ As shown in Figure 5A, the CDTM facilitated high-resolution in vivo FI at the tumor site, demonstrating its tumor targeting ability through the EPR effect. However, the positively charged CTM exhibited relatively low FI at the tumor site and the highest FI at the liver, which was consistent with previous reports on the effects of surface charge on biodistribution. The free Ce6 exhibited no FI at the tumor site. At $24 \mathrm{~h}$ post injection, the tumors and main organs (liver, spleen, kidney, heart, and lung) were excised for ex vivo fluorescence imaging to investigate the tissue distribution of nanoparticles (Figure 5B). As expected, the accumulation of 


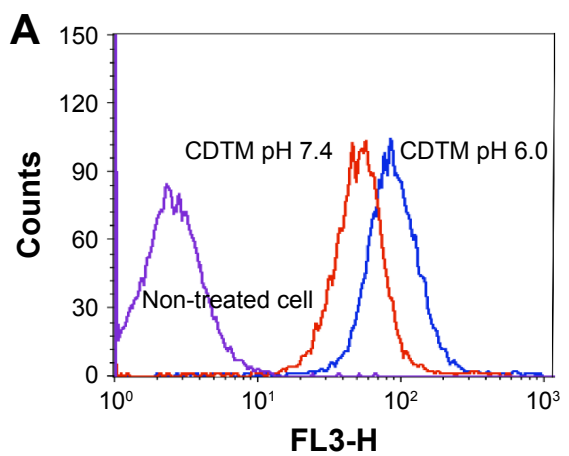

D

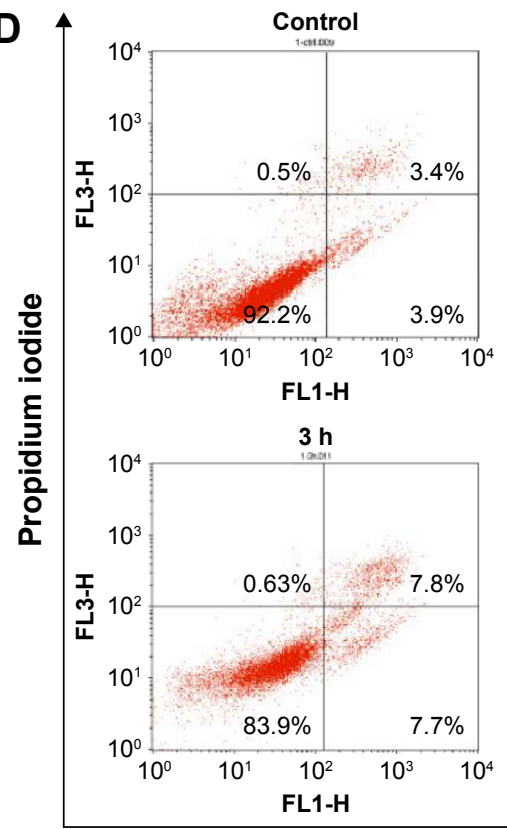

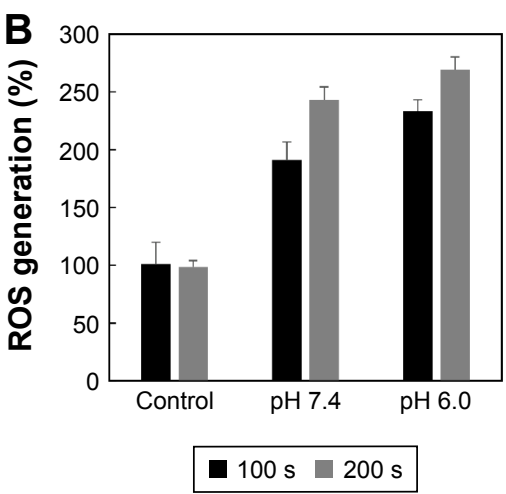
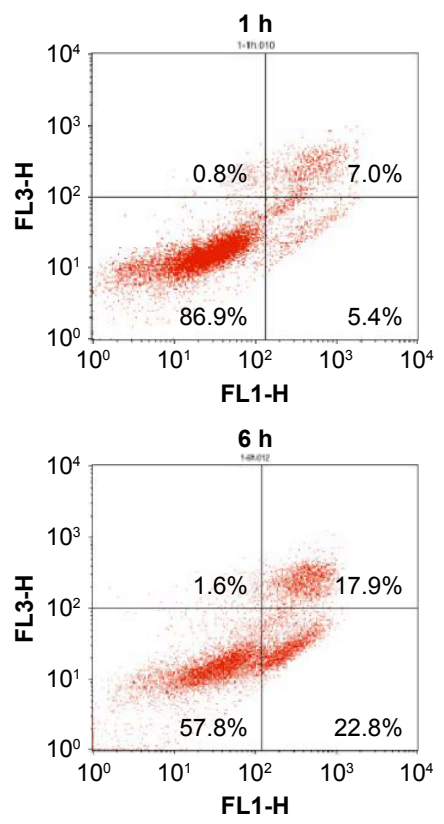

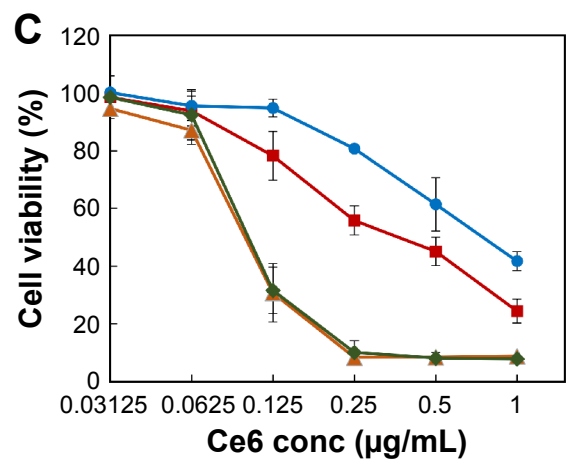

Ce6 conc $(\mu \mathrm{g} / \mathrm{mL})$ $\rightarrow$ Free $\mathrm{Ce} 6 \mathrm{pH} 6.0 \rightarrow$ Free $\mathrm{Ce} 6 \mathrm{pH} 7.4$

Annexin V FITC
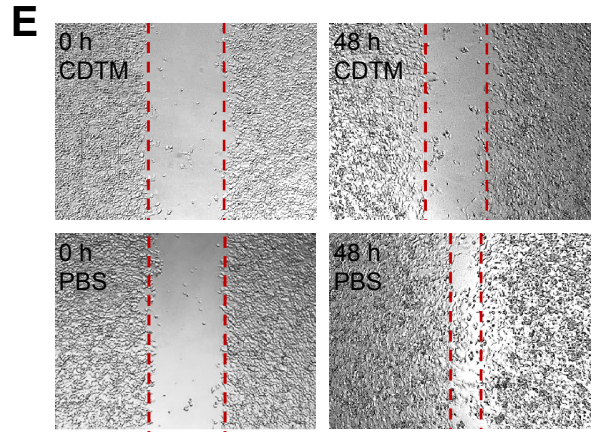

Figure 4 (A) Cellular uptake of $\mathrm{Ce} 6$ in $\mathrm{KB}$ cells at different $\mathrm{pH}$ after a $2 \mathrm{~h}$ incubation. (B) ROS generation in KB cells treated with CDTM after irradiation with light dose $5.2 \mathrm{~mW} / \mathrm{cm}^{2}$ for $100 \mathrm{~s}$ or $200 \mathrm{~s}$. (C) Viability of KB cells treated with samples at different $\mathrm{pH}$. (D) Flow cytometric analysis of time-dependent apoptosis and necrosis in $\mathrm{KB}$ cells using annexin $\mathrm{V}$ FITC and propidium iodide double staining. The KB cells were incubated with CDTM (equivalent Ce6 I $\mu g / \mathrm{mL}$ ) for $4 \mathrm{~h}$, then irradiated with $5.2 \mathrm{~mW} / \mathrm{cm}^{2}$ for $100 \mathrm{~s}$ and harvested after I, 3, 6, and $24 \mathrm{~h}(\mathrm{E})$. Wound healing assay to evaluate the migration of KB cells treated with CDTM and PBS (Ce6 $\left.=0.25 \mu \mathrm{g} / \mathrm{mL}\right)$. Abbreviations: CDTM, PEG-PLL(-g-Ce6 and DMA)-PLA micelles; Ce6, chlorin e6; ROS, reactive oxygen species.

CDTM in the tumor site was significantly higher than in the other organs, which was comparable to that observed after the administration of CTM and free Ce6. The FI ratios of the tumor to liver (FI in tumor/FI in liver) for free Ce6, CTM, and
CDTM were 1.10, 1.01, and 3.02, respectively (Figure 5C). Although the CDTM was accumulated in liver, the toxicity could be minimized because the cell damage could be exerted by locally irradiating light to target sites. 
A
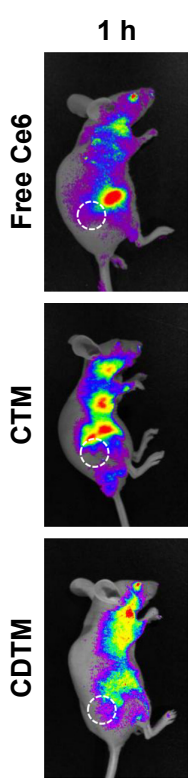

$3 \mathrm{~h}$
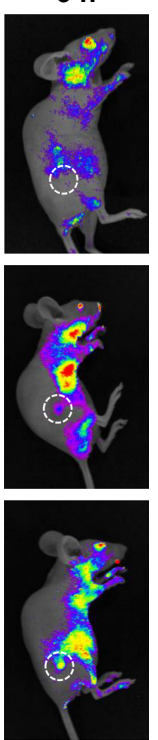

$6 \mathrm{~h}$
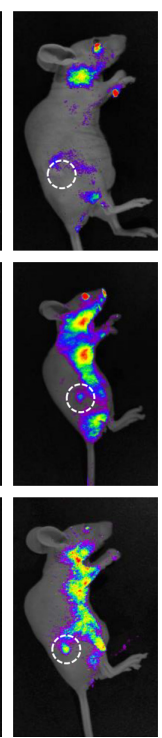

$24 \mathrm{~h}$
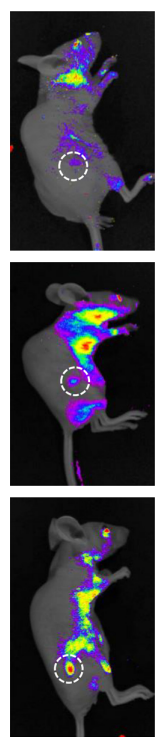

B
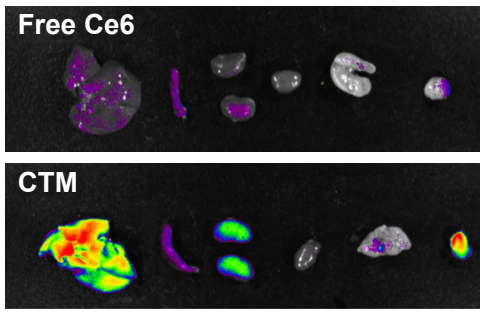

CDTM

38

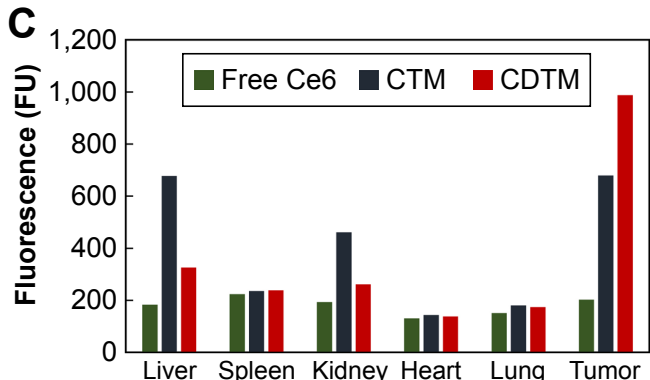

Figure 5 In vivo non-invasive fluorescent imaging of free Ce6, CTM, and CDTM injected intravenously into KB tumor-bearing nude mice.

Notes: The accumulation of CDTM at the tumor site was significantly higher than in the organs, which was comparable to that observed after the administration of CTM and free Ce6. (A) Whole body imaging at given time points after administered via tail vein injection and (B) fluorescence images of isolated liver, spleen, kidney, heart, lung and tumor after $24 \mathrm{~h}$ post injection. (C) Quantitative fluorescence intensities of tumors and main organs. The $\mathrm{Fl}$ ratios of the tumor to liver (FI in tumor/Fl in liver) for free Ce6, CTM, and CDTM were I.10, I.01, and 3.02, respectively.

Abbreviations: CDTM, PEG-PLL(-g-Ce6 and DMA)-PLA micelles; Ce6, chlorin e6; CTM, PEG-PLL(-g-Ce6)-PLA micelles; Fl, fluorescence intensity.

\section{In vivo efficacy of CDTM}

CDTM was designed with the assumption that a platform with charge-reversible properties could be used in vivo and would maintain a long circulation time with enhanced cellular uptake at the tumor site, resulting in improved anti-cancer therapeutic efficacy. Figure $6 \mathrm{~A}$ shows the tumor volume regression of $\mathrm{KB}$ tumor-bearing nude mice treated with the CDTM, CTM, free Ce6, and PBS. The data confirmed that
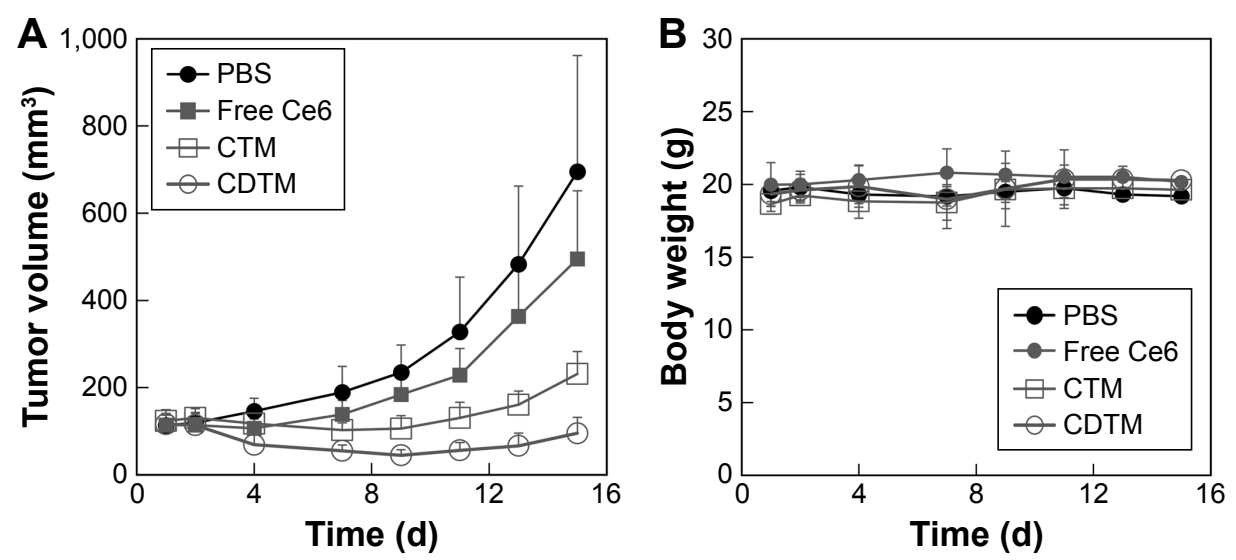

\begin{tabular}{|c|c|c|c|c|}
\hline & PBS & Free Ce6 & CTM & CDTM \\
\hline Day 1 & 0 & 0 & 0 & 0 \\
\hline Day 15 & & 0 & 0 & 0 \\
\hline
\end{tabular}

Figure 6 (A) In vivo anticancer efficacy and (B) body weight changes in KB tumor-bearing BALB/c nude mice after intravenous administration of PBS, free Ce6, CTM, and CDTM. (C) The optical images of tumor-bearing mice at day I and day I5.

Abbreviations: CDTM, PEG-PLL(-g-Ce6 and DMA)-PLA micelles; Ce6, chlorin e6; CTM, PEG-PLL(-g-Ce6)-PLA micelles. 

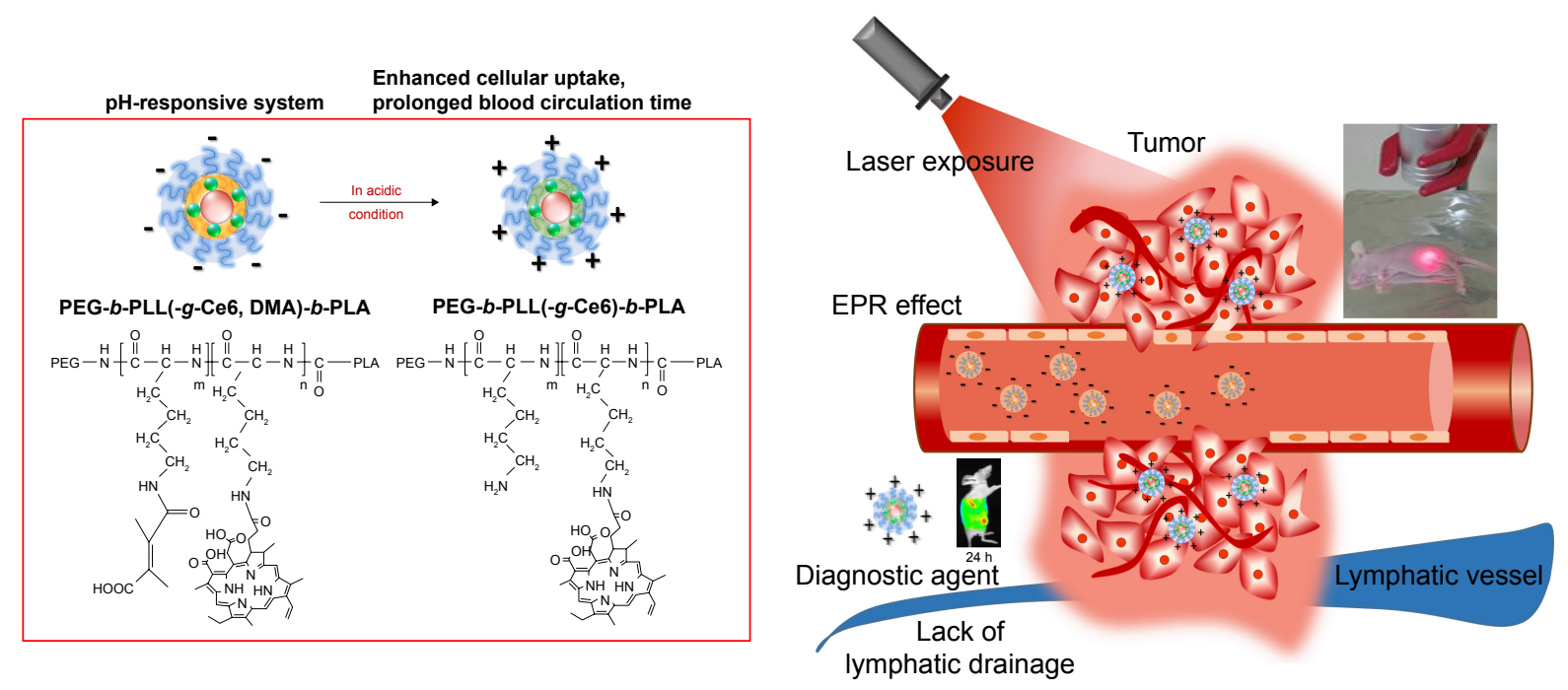

Figure 7 Schematic representation depicting the main concept of charge-reversible PDT.

Abbreviations: Ce6, chlorin e6; DMA, 2,3-dimethyl maleic anhydride; EPR, enhanced permeability and retention; PDT, photodynamic therapy; PEG, poly(ethylene glycol); PLA, poly(lactic acid); PLL, poly(L-lysine).

CDTM significantly inhibited the growth of KB tumors. The tumor volume in the nude mice treated with CDTM was $\sim 2.4$ times smaller than in those treated with CTM due to its surface charge reversibility. Although the free $\mathrm{Ce} 6$ showed the highest phototoxicity in in vitro conditions, there were no significant differences in tumor volume changes between the PBS and free Ce6 groups in vivo due to lack of tumor targeting ability of free Ce6..$^{36,45,46}$ No dramatic weight change was observed in the mice treated with any sample, indicating no apparent systemic toxicity (Figure 6B). The optical images of tumor-bearing mice at days 0 and 15 were presented in Figure $6 \mathrm{C}$ to visualize the tumor growth inhibition.

\section{Conclusion}

In this study, PEG-PLL(-g-Ce6 and DMA)-PLA triblock copolymer was synthesized and evaluated for potential use in PDT as a surface charge-reversible nanoplatform. The proposed concepts related to CDTM are presented in Figure 7. The CDTM was formed by a self-assembly process and exhibited $\mathrm{pH}$-dependent properties, such as surface charge conversion and enhanced cytotoxicity at acidic $\mathrm{pH}$ (in comparison with the results at physiologic $\mathrm{pH}$ ). The surface charge-reversible CDTM showed improved tumor targeting ability and enhanced antitumor activity in in vivo conditions compared with CTM and free Ce6. These findings demonstrate the feasibility of a $\mathrm{pH}$-sensitive PDT agent for cancer treatment. Especially, compared to other systems, the PEG-PLL(-g-Ce6, DMA)-PLA nanoparticles could form a multilayered structure and it is anticipated to have the potential to incorporate multiple drugs with sequential drug release property for use in combination drug therapy. We believe that this novel surface charge-reversible nanoplatform will allow new applications for advanced tumor therapy.

\section{Acknowledgments}

This work was supported by National Research Foundation of Korea (NRF) grants funded by the Korea government (MSIP) (NRF-2014R1A2A1A11050094 and NRF-2015R1A5A1008958) and a grant (16173MFDS542) from the Ministry of Food and Drug Safety in 2016.

\section{Disclosure}

The authors report no conflicts of interest in this work.

\section{References}

1. Brown SB, Brown EA, Walker I. The present and future role of photodynamic therapy in cancer treatment. Lancet Oncol. 2004;5(8): 497-508.

2. Dolmans DE, Fukumura D, Jain RK. Photodynamic therapy for cancer. Nat Rev Cancer. 2003;3(5):380-387.

3. Tran TH, Thapa RK, Nguyen HT, Choi HG. Combined phototherapy in anti-cancer treatment: therapeutics design and perspectives. J Pharm Investig. 2016;46(6):505-517.

4. Hopper C. Photodynamic therapy: a clinical reality in the treatment of cancer. Lancet Oncol. 2000;1(4):212-219.

5. Huang Z. A review of progress in clinical photodynamic therapy. Technol Cancer Res Treat. 2005;4(3):283-293.

6. Benachour H, Sève A, Bastogne T, et al. Multifunctional peptideconjugated hybrid silica nanoparticles for photodynamic therapy and MRI. Theranostics. 2012;2(9):889-904.

7. Celli JP, Spring BQ, Rizvi I, et al. Imaging and photodynamic therapy: mechanisms, monitoring, and optimization. Chem Rev. 2010;110(5): 2795-2838.

8. Detty MR, Gibson SL, Wagner SJ. Current clinical and preclinical photosensitizers for use in photodynamic therapy. J Med Chem. 2004; 47(16):3897-3915. 
9. Barth BM, I Altinoğlu E, Shanmugavelandy SS, et al. Targeted indocyanine-green-loaded calcium phosphosilicate nanoparticles for in vivo photodynamic therapy of leukemia. ACS Nano. 2011;5(7): 5325-5337.

10. Choi JH, Lee YJ, Kim D. Image-guided nanomedicine for cancer. J Pharm Investig. 2017;47(1):51-64.

11. Lucky SS, Soo KC, Zhang Y. Nanoparticles in photodynamic therapy. Chem Rev. 2015;115(4):1990-2042.

12. Tian J, Ding L, Xu HJ, et al. Cell-specific and pH-activatable rubyrinloaded nanoparticles for highly selective near-infrared photodynamic therapy against cancer. J Am Chem Soc. 2013;135(50):18850-18858.

13. Wang $\mathrm{C}$, Cheng $\mathrm{L}$, Liu $\mathrm{Y}$, et al. Imaging-guided $\mathrm{pH}$-sensitive photodynamic therapy using charge reversible upconversion nanoparticles under near-infrared light. Adv Funct Mater. 2013;23(24): 3077-3086.

14. Park SY, Baik HJ, Oh YT, Oh KT, Youn YS, Lee ES. A smart polysaccharide/drug conjugate for photodynamic therapy. Angew Chem Int Ed Engl. 2011;50(7):1644-1647.

15. Yu B, Zhang Y, Zheng W, Fan C, Chen T. Positive surface charge enhances selective cellular uptake and anticancer efficacy of selenium nanoparticles. Inorg Chem. 2012;51(16):8956-8963.

16. Kamimura M, Kim JO, Kabanov AV, Bronich TK, Nagasaki Y. Block ionomer complexes of PEG-block-poly(4-vinylbenzylphosphonate) and cationic surfactants as highly stable, $\mathrm{pH}$ responsive drug delivery system. J Control Release. 2012;160(3):486-494.

17. Lim C, Sim T, Hoang NH, Oh KT. A stable nanoplatform for antitumor activity using PEG-PLL-PLA triblock co-polyelectrolyte. Colloids Surf B Biointerfaces. 2017;153:10-18.

18. Fröhlich E. The role of surface charge in cellular uptake and cytotoxicity of medical nanoparticles. Int J Nanomedicine. 2012;7:5577-5591.

19. He C, Hu Y, Yin L, Tang C, Yin C. Effects of particle size and surface charge on cellular uptake and biodistribution of polymeric nanoparticles. Biomaterials. 2010;31(13):3657-3666.

20. Tang S, Meng Q, Sun H, et al. Dual pH-sensitive micelles with chargeswitch for controlling cellular uptake and drug release to treat metastatic breast cancer. Biomaterials. 2017;114:44-53.

21. Du JZ, Du XJ, Mao CQ, Wang J. Tailor-made dual pH-sensitive polymer-doxorubicin nanoparticles for efficient anticancer drug delivery. J Am Chem Soc. 2011;133(44):17560-17563.

22. Lim C, Youn YS, Lee KS, et al. Development of a robust $\mathrm{pH}$-sensitive polyelectrolyte ionomer complex for anticancer nanocarriers. Int $J$ Nanomedicine. 2016;11:703-713.

23. Song HT, Hoang NH, Yun JM, et al. Development of a new tri-block copolymer with a functional end and its feasibility for treatment of metastatic breast cancer. Colloids Surf B Biointerfaces. 2016;144:73-80.

24. Ku EB, Oh KT, Youn YS, Lee ES. Mitochondria-selective photodynamic tumor therapy using globular PEG nanoparticles. Macromol Res. 2016;24(7):634-639.

25. Lee JM, Kwag DS, Youn YS, Lee ES. Gas-forming liposomes prepared using a liposomal magnetoporation method. Colloids Surf B Biointerfaces. 2017;155:209-214.

26. Yarrow JC, Perlman ZE, Westwood NJ, Mitchison TJ. A high-throughput cell migration assay using scratch wound healing, a comparison of image-based readout methods. BMC Biotechnol. 2004;4(1):21.

27. Hur K, Toiyama Y, Takahashi M, et al. MicroRNA-200c modulates epithelial-to-mesenchymal transition (EMT) in human colorectal cancer metastasis. Gut. 2013;62(9):1315-1326.
28. Deng H, Zhao X, Liu J, et al. Reactive oxygen species (ROS) responsive PEG-PCL nanoparticles with $\mathrm{pH}$-controlled negative-to-positive charge reversal for intracellular delivery of doxorubicin. J Mater Chem B. 2015;3(48):9397-9408.

29. Lee BR, Oh KT, Oh YT, et al. A novel pH-responsive polysaccharidic ionic complex for proapoptotic day-(KLAKLAK)2 peptide delivery. Chem Commun (Camb). 2011;47(13):3852-3854.

30. Stebounova LV, Guio E, Grassian VH. Silver nanoparticles in simulated biological media: a study of aggregation, sedimentation, and dissolution. J Nanopart Res. 2011;13(1):233-244.

31. Bagwe RP, Hilliard LR, Tan W. Surface modification of silica nanoparticles to reduce aggregation and nonspecific binding. Langmuir. 2006;22(9):4357-4362.

32. Freitas C, Müller RH. Effect of light and temperature on zeta potential and physical stability in solid lipid nanoparticle (SLN ${ }^{\mathrm{TM}}$ ) dispersions. Int J Pharm. 1998;168(2):221-229.

33. Otsuka H, Nagasaki Y, Kataoka K. PEGylated nanoparticles for biological and pharmaceutical applications. Adv Drug Deliv Rev. 2003; 55(3):403-419.

34. Diwan M, Park TG. Pegylation enhances protein stability during encapsulation in PLGA microspheres. J Control Release. 2001;73(2-3): 233-244.

35. Park $\mathrm{H}, \mathrm{Na} \mathrm{K}$. Conjugation of the photosensitizer Chlorin e6 to pluronic F127 for enhanced cellular internalization for photodynamic therapy. Biomaterials. 2013;34(28):6992-7000.

36. Lee DJ, Park GY, Oh KT, et al. Multifunctional poly (lactide-coglycolide) nanoparticles for luminescence/magnetic resonance imaging and photodynamic therapy. Int J Pharm. 2012;434(1-2):257-263.

37. Li F, Na K. Self-assembled chlorin e6 conjugated chondroitin sulfate nanodrug for photodynamic therapy. Biomacromolecules. 2011;12(5): 1724-1730.

38. Chambers AF, Groom AC, MacDonald IC. Metastasis: dissemination and growth of cancer cells in metastatic sites. Nat Rev Cancer. 2002; 2(8):563-572.

39. Chaffer CL, Weinberg RA. A perspective on cancer cell metastasis. Science. 2011;331(6024):1559-1564.

40. Li Y, Yu Y, Kang L, Lu Y. Effects of chlorin e6-mediated photodynamic therapy on human colon cancer SW480 cells. Int J Clin Exp Med. 2014; 7(12):4867-4876.

41. Yang TH, Chen CT, Wang CP, Lou PJ. Photodynamic therapy suppresses the migration and invasion of head and neck cancer cells in vitro. Oral Oncol. 2007;43(4):358-365.

42. Park SY, Oh KT, Oh YT, Oh NM, Youn YS, Lee ES. An artificial photosensitizer drug network for mitochondria-selective photodynamic therapy. Chem Commun (Camb). 2012;48(19):2522-2524.

43. Bremer C, Ntziachristos V, Weissleder R. Optical-based molecular imaging: contrast agents and potential medical applications. Eur Radiol. 2003;13(2):231-243.

44. Zhu S, Hong M, Zhang L, Tang G, Jiang Y, Pei Y. PEGylated PAMAM dendrimer-doxorubicin conjugates: in vitro evaluation and in vivo tumor accumulation. Pharm Res. 2010;27(1):161-174.

45. Oh NM, Oh KT, Youn YS, et al. Poly(1-aspartic acid) nanogels for lysosome-selective antitumor drug delivery. Colloids SurfB Biointerfaces. 2013;101:298-306.

46. Lee JO, Oh KT, Kim D, Lee ES. pH-sensitive short worm-like micelles targeting tumors based on the extracellular pH. J Mater Chem B. 2014; 2(37):6363-6370. 


\section{Supplementary material}
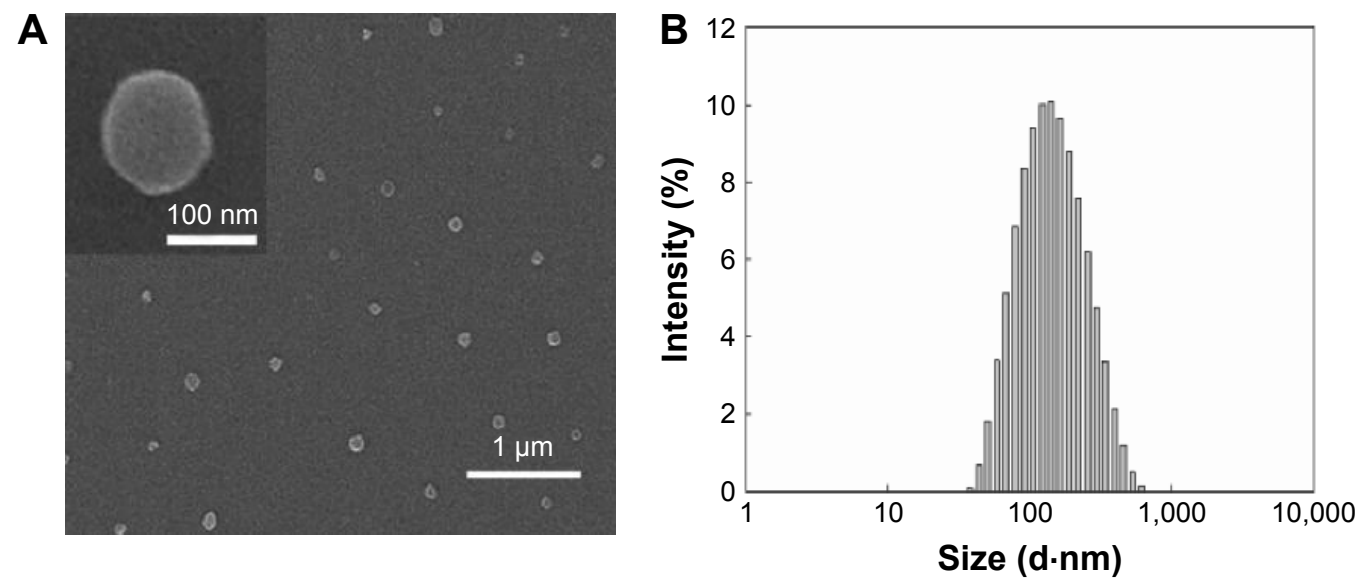

Figure SI Characterization of CDTM. FE-SEM imaging (A) and particle size distribution (B) of CDTM at pH 7.4.

Notes: The morphology of CDTM at $\mathrm{pH} 7.4$ obtained from FE-SEM revealed regular spherical particles with a narrow size distribution. Magnification $\times 20,000$.

Abbreviations: CDTM, PEG-PLL(-g-Ce6 and DMA)-PLAmicelles; FE-SEM, field emission scanning electron microscope.

\section{Publish your work in this journal}

The International Journal of Nanomedicine is an international, peerreviewed journal focusing on the application of nanotechnology in diagnostics, therapeutics, and drug delivery systems throughout the biomedical field. This journal is indexed on PubMed Central, MedLine, CAS, SciSearch ${ }^{\circledR}$, Current Contents ${ }^{\circledR} /$ Clinical Medicine,
Journal Citation Reports/Science Edition, EMBase, Scopus and the Elsevier Bibliographic databases. The manuscript management system is completely online and includes a very quick and fair peer-review system, which is all easy to use. Visit http://www.dovepress.com/ testimonials.php to read real quotes from published authors. 